\title{
Saberes ancestrales sobre el uso de flora y fauna en la comunidad indígena Tikuna de Cushillo Cocha, zona fronteriza Perú-Colombia-Brasil
}

\section{Ancestral knowledge about the use of flora and fauna in the indigenous community Tikuna from Cushillo Cocha, border with Peru-Colombia-Brazil}

\section{Elsa Rengifo-Salgado ${ }^{1 *}$, Sandra Rios-Torres ${ }^{1}$, Lizardo Fachín Malaverri ${ }^{1}$ y Gabriel Vargas-Arana ${ }^{2}$}

1 Instituto de Investigaciones de la Amazonía Peruana, Av. Abelardo Quiñones km 2.5, lquitos, Perú.

2 Universidad Científica del Perú, Av. Abelardo Quiñones \# 2500, Iquitos, Perú.

* Autor para correspondencia.

Email Elsa Rengifo-Salgado: erengifo@iiap.org.pe

Email Sandra Rios-Torres: srios@iiap.org.pe

Email Lizardo Fachín Malaverri: Ifachin@iiap.org.pe

Email Gabriel Vargas-Arana: gvargas@ucp.edu.pe

\section{Resumen}

En el presente estudio se reportan los conocimientos ancestrales indígenas (etnoconocimientos) de la comunidad indígena Tikuna de Cushillo Cocha. La comunidad está ubicada en la provincia de Ramón Castilla, al nororiente de la Región Loreto, Perú. La recolección de datos se realizó a través de talleres participativos, entrevistas y recorridos biológicos para realizar la recolectar de especies vegetales, observar a animales in situ, acompañados por los pobladores con experiencia y conocimiento de su territorio. Un total de 247 especies de flora y fauna fueron registradas. En etnobotánica se identificaron 101 especies, en siete categorías de uso asignados por los pobladores, siendo las categorías de alimentación y medicinal las de mayor representatividad. Para el caso de etnozoología se identificaron 146 especies, que son utilizadas en seis categorías, siendo las de mayor relevancia las categorías de alimentación y mascotas. Se evidenció que el bosque es la fuente principal para la alimentación, cura de enfermedades, elaboración de artesanías, como mascotas para la venta y plantas como ornamentales, todo esto con importante aceptación por parte de la población indígena.

Palabras clave: Usos tradicionales; Tikuna; Cushillo Cocha; etnobotánica; etnozoología.

\section{Abstract}

In the present study, ancestral indigenous knowledge (ethno-knowledge) of the indigenous community "Tikuna from Cushillo Cocha" is reported. The community is located in the province of Ramón Castilla, northeast of Loreto Region, Peru. Data was collected through participatory workshops, interviews and biological expeditions in order to collect plant species, observe animals and identify them in situ, always accompanied by local people considered them with experience and knowledge of their territory. A total of 247 species of flora and fauna were registered. In ethnobotany, 101 species were identified, grouped in seven categories of assigned uses by locals, being food and medicinal categories the most representative. For the case of ethnozoology, 146 species were identified, which are used in six categories, being food and pet categories the most relevant. It was evidenced that the forest is the main source for food, medical supplies, handicrafts, animals as pets to be sold and plants as ornamental, all these with important acceptance by the indigenous population.

Keywords: Traditional uses; Tikuna; Cushillo Cocha; ethnobotany; ethnozoology.

Citación :

Rengifo-Salgado E., S. Rios-Torres, L. Fachín Malaverri \& G. VargasArana. 2017. Saberes ancestrales sobre el uso de flora y fauna en la comunidad indígena Tikuna de Cushillo Cocha, zona fronteriza PerúColombia-Brasil. Revista peruana de biología 24(1): 067 - 078 (Marzo 2017). doi: http://dx.doi.org/10.15381/rpb.v24i1.13108
Fuentes de financiamiento: Instituto de Investigaciones de la Amazonia Peruana- Meta 28-2014-Proyecto Recuperación de conocimientos etnobiológicos para la conservación de la biodiversidad.

Información sobre los autores:

ERS realizó el diseño, ejecución y análisis de la investigación, redacción inicial del artículo; SRT colaboró con el análisis de la investigación as como la corrección del artículo; LFM aportó su experiencia en SIG con la elaboración del mapa de ubicación, traducción al inglés y corrección del artículo; GVA realizó el análisis de los datos, procesamiento de los resultados y apoyó en la redacción y corrección del artículo.

Los autores, aportaron de su experticia sin incurrir en conflicto de intereses. $\begin{array}{ll}\text { Presentado: } & 22 / 06 / 2016 \\ \text { Aceptado: } & 06 / 11 / 2016\end{array}$

Publicado online: 15/04/2017 


\section{Introducción}

Los conocimientos tradicionales son un componente esencial en el vivir diario de millones de personas en los países en desarrollo, tanto en las comunidades indígenas como en las mestizas. Las comunidades indígenas han utilizado los conocimientos tradicionales durante siglos, bajo sus leyes locales, sus costumbres y sus tradiciones, que han sido transmitidos y han ido evolucionando de generación en generación. Estos conocimientos tradicionales han jugado, y juegan aún, un papel importante en áreas vitales como la seguridad alimentaria, el desarrollo agrícola y los tratamientos medicinales (Correa 2001).

El papel de la etnobiología radica en entender y reconocer por una parte que la sabiduría tradicional y local puede caracterizar y aportar en un proceso de desarrollo sostenible y sostenido, y por otro lado que las culturas que nutren de conocimiento a las etnociencias están desapareciendo de manera continua y creciente, en particular por la pérdida de su hábitat, y también por la pobreza y miseria en la que se ven sumidas gracias al "desarrollismo", que las empuja a convertirse a su manera o desaparecer. Los conocimientos de las comunidades indígenas y locales, son una acumulación dinámica, son patrimonio colectivo, son un sistema organizado de investigación y descubrimientos, con experiencias milenarias de practicar, mirar, aprender, probar, asumir y transformar esa realidad (Escobar Berón 2002).

Uno de los aportes de los conocimientos ancestrales es la medicina tradicional, que es un conjunto de conocimientos y prácticas, que tienen como fundamento el saber médico ancestral de una población. Es una práctica que se trasmite por la tradición familiar o comunitaria, que tiene sus propios agentes de salud y sus ideas específicas sobre la enfermedad y la curación. Es el saber del pueblo (folklore) que se puede identificar en los campos y ciudades de la Amazonía (Estrella 1995).

En América Latina, el uso de animales también representa una alternativa a las prácticas médicas oficiales en las zonas rurales, y también se ha convertido en parte de la medicina popular urbana. Se han registrado que al menos 584 animales se utilizan con fines medicinales en América Latina, lo que subraya su importancia como alternativa terapéutica en la región (Alves \& Alves 2011).

En el Perú, la riqueza botánica se refleja en la diversidad de usos de plantas: cerca de 5000 especies de plantas son utilizadas por la población peruana para la alimentación, la medicina, adornos, construcción, forraje, colorantes, toxinas, y la leña, entre otros usos. Por encima de todo, las comunidades rurales dependen en gran medida de las plantas para su subsistencia (Bragg Egg 1999). Por otra parte, estudios realizados mencionan que, en la Amazonía peruana, 15 grupos lingüísticos se han reportado extintos en un lapso de 75 años desde 1900 (Ribeiro y Wise 1979) y que otros 13 grupos lingüísticos se encuentran en proceso de extinción (Solis 2003). Los censos poblacionales de los años 1970 y 1993 indican una disminución progresiva de la diversidad cultural en un 15\% (Varesse 1983; INEI 1993). De este modo, los conocimientos indígenas acumulados y transmitidos oralmente o dentro de cualquier expresión cultural durante milenios, podrían desaparecer irremediablemente en menos de dos generaciones (Alexiades 1985)

$\mathrm{El}$ reconocimiento de los pueblos indígenas como autores y gestores activos de conocimiento, en vez de considerarlos como simples fuentes de información, está tomando fuerza en una economía basada en la conservación y aprovechamiento de los recursos renovables. El hecho de que el 65\% de la trasmisión del conocimiento tradicional sea todavía oral, ejemplifica la importancia que tiene la preservación cultural de los pueblos indígenas (Bajak 2014)

Los Tikuna, son una etnia que se localiza generalmente en las riberas de pequeños ríos que desembocan en el Amazonas, esto abarca algo más de mil kilómetros del alto amazonas, entre Brasil, Perú y Colombia. Los Tikuna son el grupo indígena con la mayor población de toda la cuenca Amazónica (López 2002), ascendiendo a un aproximado de 30000 habitantes, distribuidos de la siguiente manera: el $55 \%$ en 58 asentamientos en los municipios de Tabatinga, Japurá, Sao Paulo de Olivenca, San Antonio do Icá, en territorio brasileño, el 27\% en 27 asentamientos en los municipios de Leticia y Puerto Narińo en territorio colombiano, el $18 \%$ en 15 asentamientos en las provincias de Ramón Castilla, Yavarí y Putumayo en territorio peruano (Acosta 2001). En la Amazonía peruana, si bien el promedio poblacional se ubica en 162 individuos por comunidad, se observa una gran concentración en la comunidad de Cushillo Cocha (Mayor \& Bodmer 2009).

Desde el punto de vista del conocimiento de los saberes ancestrales, pocos son los estudios que se han realizado sobre la comunidad Tikuna en la Amazonía peruana (Valadeau 2009), a diferencia de la Amazonía colombiana y brasileńa, donde abarcan temas sobre usos tradicionales de plantas medicinales, pesca, su interacción con la naturaleza para su supervivencia, etc. (Quintana 2012, Vieco y Oyuela 1999, Hammond et al. 1995, Rodrigues 2006, Carlini et al. 2006).

$\mathrm{El}$ presente trabajo tuvo como objetivo rescatar, registrar y difundir los etnoconocimientos referidos al uso y manejo de los recursos naturales de flora y fauna en la comunidad indígena Tikuna de Cushillo Cocha en la provincia de Ramón Castilla al nororiente de la Región Loreto, Perú. Este trabajo constituye el primer aporte de datos referidos al uso popular de plantas y animales, con diferentes fines para esta comunidad indígena en la Amazonía peruana, que están desapareciendo debido a diversos factores, entre ellos el ingreso de productos manufacturados externos y a la visión globalizante y comercial, de instituciones públicas y privadas presentes en la zona.

\section{Material y métodos}

Área de Estudio.- La comunidad indígena Tikuna de Cushillo Cocha ( $3^{\circ} 56^{\prime} 25^{\prime}$ 'S, $\left.70^{\circ} 27^{\prime} 11^{\prime \prime} \mathrm{W}\right)$ se encuentra asentada a orillas del lago Cushillo Cocha (el Lago es un afluente del río Amazonas), en la provincia Ramón Castilla, Región Loreto, Perú (Figura 1).

Esta comunidad está habitada por familias pertenecientes al pueblo indígena Tikuna, pueblo cuya lengua ha sido clasificada dentro de la familia etnolingüística del mismo nombre. La comunidad aún conserva su lengua materna, así como sus mitos, leyendas, costumbres y folclore. Las leyendas, el empleo de las medicinas nativas, los quehaceres de la casa, la construcción de casa y canoas, etc., son transmitidos de padres a hijos (Riveiro $\&$ Wise 1979). En la actualidad la comunidad cuenta con 2720 habitantes, distribuidos en 735 viviendas.

La comunidad de Cushillo Cocha se encuentra inscrita en los registros de comunidades nativas mediante Resolución $\mathrm{N}^{\circ}$ 


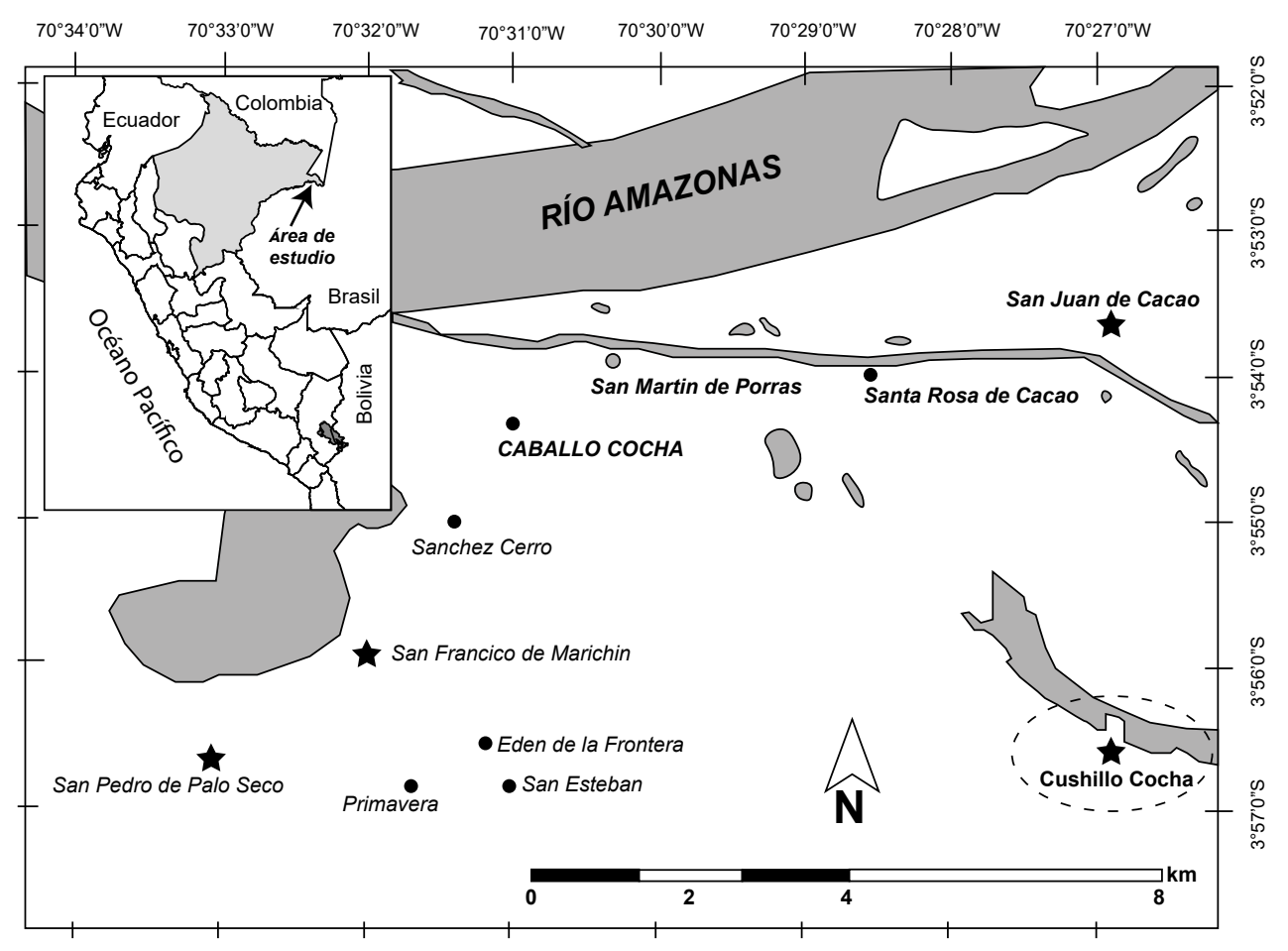

Figura 1. Comunidad nativa Tikuna de Cushillo Cocha

124-75-OAE-ORAMSV del 26 de junio del año 1975, con un área de 13253 hectáreas (MINCETUR 2009).

Metodología.- La investigación se llevó a cabo el año 2014, el ingreso a la zona de estudio (comunidad) se realizó en tres oportunidades, cada una duro siete días, y en todas se realizaron las actividades con participación activa de los pobladores.

El proceso de recuperación de los conocimientos y la obtención de los datos en la comunidad, se realizó inicialmente con la visita a las autoridades, donde se les expuso sobre nuestro objetivo y solicitamos su autorización para realizar la investigación y actividades. Como apertura se realizó un taller con los pobladores convocados por las autoridades, posteriormente entrevistas in situ, recorridos botánicos en sus chacras y huertos. Los talleres fueron interactivos, describiendo las plantas y animales que conocen, cómo las utilizan y cuáles tienen en sus alrededores. Las entrevistas complementaron la información de los talleres, se aplicó a hombres y mujeres adultas familiarizadas con la flora y fauna existente en la zona.

Las especies fueron identificadas por los pobladores mediante soporte visual, con la ayuda de láminas con figuras de plantas y animales amazónicos. Fueron utilizadas como guías las siguientes referencias: Martín (2009), López-Wong (2013); GagliardiUrrutia (2013); del-Águila-Chávez (2013), Rengifo-Salgado (2013) y Mejía (2013). Los pobladores indicaron los usos de las especies y su categorizaron se realizó según estos usos.

Los nombres de los animales y plantas fueron traducidas al idioma Tikuna por el Sr. Gustavo Pashia Moreno, oriundo de la comunidad nativa y conocedor de las especies de flora y fauna, quien confirmó algunas especies respecto a lo publicado por Montes (2001).

Posteriormente, los datos recopilados fueron analizados y sistematizados en gabinete, tanto para plantas como para animales.
El proceso de identificación para las especies vegetales, fue por identificación in situ. Para el esquema de Categorías Taxonómicas de Orden y Familia, se siguió el Sistema de Clasificación de APG III (2009). No se solicitó de autorización de SERFOR, porque es un estudio donde no se colectaron muestras, solo los conocimientos de estas y se observaron in situ.

\section{Resultados}

A continuación, se presenta el análisis y la sistematización de información correspondiente a la flora y fauna.

Las entrevistas se realizaron a 60 personas, con edades entre 18 y 80 años. El 66.7\% (40 personas) del sexo masculino y el $33.3 \%$ (20 personas) del sexo femenino. Se registraron un total de 247 especies entre flora y fauna, 101 especies de plantas (etnobotánica) y 146 especies de animales (etnozoología).

Las 101 especies de plantas registradas pertenecen a 92 géneros y 40 familias. Estas especies se agruparon en siete categorías de uso: Alimenticio (A), Artesanía (Art.), Combustible (Comb.), Construcción (C), Herramienta (H), Medicinal (M) y Tinte (T) (Tabla 1).

Las familias de plantas más representadas fueron: Fabaceae (13 especies), Arecaceae (11 especies), Euphorbiaceae (7 especies), Moraceae (6 especies), Annonaceae (5 especies), Apocynaceae ( 4 especies) y Rubiaceae ( 4 especies), mientras que las familias restantes se encuentran representadas por 1, 2 o 3 especies.

Las categorías de uso con mayor número de especies de plantas fueron: alimentación con 46 especies y medicinal con 41. Con un solo uso se registraron 69 especies, con dos usos 30 especies, con tres usos una especie y con cuatro usos también una sola especie. La especie con mayor número de usos fue $A s$ trocaryum chambira (chambira), que es usada para alimentación, artesanía, herramienta y como medicina. En la Tabla 2 se detallan 
Tabla 1. Especies vegetales usadas por los pobladores de la comunidad Tikuna de Cushillo Cocha

\begin{tabular}{|c|c|c|c|c|c|}
\hline $\mathbf{N}^{\circ}$ & Nombre común & Nombre Tikuna & Especie & Familia & Usos \\
\hline 1 & Achiote* & Üxta & Bixa orellana $\mathrm{L}$ & Bixaceae & $\mathrm{T}$ \\
\hline 2 & Aguaje & Texma & Mauritia flexuosa L. f & Arecaceae & $\mathrm{A}, \mathrm{C}$ \\
\hline 3 & Ají* & Mexe & Capsicum annuum L. & Solanaceae & M \\
\hline 4 & Algodón* & Tüx & Gossypium barbadense L. & Malvaceae & M \\
\hline 5 & Amasisa & Taxtüne & Erythrina fusca Loureiro. & Fabaceae & M \\
\hline 6 & Anacaspi & Sha & Apuleia leiocarpa (J.Vogel) J.F.Macbride & Fabaceae & $\mathrm{C}$ \\
\hline 7 & Andiroba & Aüdiruba & Carapa guianensis Aublet & Meliaceae & $\mathrm{C}$ \\
\hline 8 & Anona* & Naxú-wirina & Annona scandens var. scandens Diels & Annonaceae & A \\
\hline 9 & Añuje Caspi & Chigu Bü & Swartzia polyphylla DC & Fabaceae & Art \\
\hline 10 & Arazá* & Aracha & Eugenia stipitata subsp stipitata Mc. Vaugh & Myrtaceae & $\mathrm{A}, \mathrm{M}$ \\
\hline 11 & Arroz* & Aruchu & Oryza sativa $L$ & Poaceae & $\mathrm{A}, \mathrm{M}$ \\
\hline 12 & Azúcar huayo & Caxuta & Hymenaea reticulata Ducke & Fabaceae & $\mathrm{A}, \mathrm{C}$ \\
\hline 13 & Bellaco caspi & Maxniú & Himatanthus sucuuba (Spruce ex. Muell .Arg) Woodson & Apocynaceae & $\mathrm{C}$ \\
\hline 14 & Caballusa & Caballusa & Triumfetta althaeoides Lamarck & Tiliaceae & M \\
\hline 15 & Cacao* & Chaxpere & Theobrama cacao L. & Sterculiaceae & A \\
\hline 16 & Caguena* & - & Ayapana tripinervis (Vahl) R.M.King \& H.Rob. & Asteraceae & M \\
\hline 17 & Caimito* & Taxü & Pouteria caimito (R. \& P.) Radlkofer. & Sapotaceae & A \\
\hline 18 & Canela moena & Arupane & Ocotea javitensis (H.B.K) Pittier & Lauraceae & $\mathrm{C}$ \\
\hline 19 & Caña* & Dexne & Saccharum officinarum L. & Poaceae & A \\
\hline 20 & Caoba & Cauba & Swietenia macrophylla King & Meliaceae & $\mathrm{C}$ \\
\hline 21 & Capirona & Comechi & Calycophyllum spruceanum( Bentham) & Rubiaceae & $\mathrm{C}$ \\
\hline 22 & Castaña & Baxe & Bertholletia excelsa Humboldt \& Bonpland & Lecythidaceae & A \\
\hline 23 & Catahua & Catawa & Hura crepitans $\mathrm{L}$. & Euphorbiaceae & $\mathrm{A}, \mathrm{C}$ \\
\hline 24 & Cedrillo & Ocayiwa Chicü & Vochysia misiifolia Spruce ex. Warming & Voshysiaceae & $\mathrm{C}$ \\
\hline 25 & Cetico & Õmaúwa & Cecropia membranacea Trecul & Cecropiaceae & $\mathrm{C}, \mathrm{M}$ \\
\hline 26 & Charichuelo* & - & Garcinia macrophylla C.Martius & Clusiaceae & A \\
\hline 27 & Chambira & Naxi & Astrocaryum chambira Burret & Arecaceae & $\begin{array}{l}\text { A, Art, } \\
\mathrm{H}, \mathrm{M}\end{array}$ \\
\hline 28 & Chambira huayo & - & Astrocaryum chonta C. Martius & Arecaceae & A, $\mathrm{H}$ \\
\hline 29 & Chancapiedra & Yirutipara & Phyllanthus niruri L. & Euphorbiaceae & M \\
\hline 30 & Chicle huayo & Ngexchi & Lacmellea peruviana (Van Heurck \& Muell.Arg.) Markgraf & Apocynaceae & $\mathrm{C}$ \\
\hline 31 & Chiricsanango & Chanagu & Brunfelsia grandifolia D.Don & Solanaceae & M \\
\hline 32 & Chontaquiro & Chontaquiro & Diplotropis purpurea var. leptophylla ( Kleinhoonte)Amshoff & Fabaceae & M \\
\hline 33 & Cocona* & Bere & Solamun sessiliflorum Dunal & Solanaceae & A \\
\hline 34 & Copal blanco & Poene & Crepidospermun prancei Daly & Burseraceae & $\mathrm{C}$ \\
\hline 35 & Copaiba & - & Capaifera paupera (Herzog) Dwyer & Fabaceae & M \\
\hline 36 & Culantrillo & Chicuri & Adiantum obliquum Willd. & Pteridaceae & A \\
\hline 37 & Cunchi moena & Ngúxchi & Endlicheria krukovii (A.C.Smith) & Lauraceae & $\mathrm{C}$ \\
\hline 38 & Espintana & Chixane & Anaxagorea brachycarpa R.E.Fries & Annonaceae & $\mathrm{C}$ \\
\hline 39 & $\begin{array}{l}\text { Frejol o } \\
\text { Chiclayo* }\end{array}$ & Purutu & Vigna unguiculata (L.) Walpers & Fabaceae & $\mathrm{A}, \mathrm{M}$ \\
\hline 40 & Granadilla & Boxra & Passiflora laurifolia $\mathrm{L}$. & Passifloraceae & A \\
\hline 41 & Guanábana* & - & Annona muricata L. & Annonaceae & A \\
\hline 42 & Guaba* & Pama & Inga edulis C. Martius & Fabaceae & A \\
\hline 43 & Guacamayo caspi & Ngoxü arü naxi & Coutarea hexandra (Jacquin) Schumann & Rubiaceae & $\mathrm{T}$ \\
\hline 44 & Guayaba* & Oracha & Psidium guajava $\mathrm{L}$. & Myrtaceae & A \\
\hline 45 & Guineo* & Irú & Musa paradisiaca $\mathrm{L}$. & Musaceae & $\mathrm{A}, \mathrm{M}$ \\
\hline 46 & Guisador* & Dexpaú & Curcuma longa $\mathrm{L}$. & Zingiberaceae & A \\
\hline 47 & Huacapu & Chexe & Minquartia guianensis Aublet & Olacaceae & C, Art \\
\hline 48 & Huacapurana & Wacapu & Campsiandra angustifolia Spruce ex Bentham & Fabaceae & M \\
\hline 49 & Huaruma & Dexpe & Delostoma integrifolium D.Don & Bignoniaceae & $\mathrm{H}, \mathrm{M}$ \\
\hline 50 & Huasí & Waira & Euterpe precatoria C. Martius & Arecaceae & $\mathrm{A}, \mathrm{T}$ \\
\hline 51 & Huayruro & Muruweta & Ormosia coccinea var. subsimplex (Spruce ex Betham) Rudd & Fabaceae & C, Art \\
\hline 52 & Huito & $\overline{\mathrm{E}}$ & Genipa americana $\mathrm{L}$. & Rubiaceae & A, M \\
\hline 53 & Icoja & Icoja & Unonopsis spectabilis Diels & Annonaceae & M \\
\hline 54 & Ipururo & - & Alchornea castaneifolia (Willdenow) Adr.Jussieu. & Euphorbiaceae & M \\
\hline
\end{tabular}


Tabla 1. Continuación

\begin{tabular}{|c|c|c|c|c|c|}
\hline $\mathbf{N}^{\circ}$ & Nombre común & Nombre Tikuna & Especie & Familia & Usos \\
\hline 55 & Irapay & Coxtu & Lepidocaryum tenue C. Martius & Arecaceae & $\mathrm{A}, \mathrm{C}$ \\
\hline 56 & Jergón sacha & Ãtape arü Ĩxe & Dracontium loretense $\mathrm{K}$.Krause & Araceae & $\mathrm{A}, \mathrm{M}$ \\
\hline 57 & Leche caspi & Ngexchi & Couma macrocarpa Barbosa Rodriguez & Apocynaceae & $\mathrm{A}, \mathrm{C}$ \\
\hline 58 & Limón* & Irimawa & Citrus limon $(\mathrm{L})$ & Rutaceae & A \\
\hline 59 & Macambo* & $\mathrm{Ngu}$ & Theobroma bicolor Humboldt \& Bonpland & Malvaceae & $\mathrm{A}, \mathrm{M}$ \\
\hline 60 & Machimango & Tüxrabüne & Eschweilera gigantea (Knuth) J.F Macbride & Lecythidaceae & $\mathrm{C}$ \\
\hline 61 & Maíz* & Chawü & Zea mays $\mathrm{L}$ & Poaceae & A \\
\hline 62 & Malva & - & Malachra radiata $(\mathrm{L}.) \mathrm{L}$. & Malvaceae & M \\
\hline 63 & Mango* & Maügua & Mangifera indica $\mathrm{L}$. & Anacardiaceae & $\mathrm{A}, \mathrm{M}$ \\
\hline 64 & Marupa & Maruxpa & Simarouba amara Aublet & Simaroubaceae & $\mathrm{C}$ \\
\hline 65 & Mishquipanga & - & Renealmia nicolaioides Loesener & Zingiberaceae & $\mathrm{T}$ \\
\hline 66 & Naranja* & - & Citrus sinensis $\mathrm{L}$. & Rutaceae & A \\
\hline 67 & Oje & Poxta & Ficus insipida Wildenow & Moraceae & Art, $\mathrm{M}$ \\
\hline 68 & Paico* & Maturuchi & Dysphania ambrosioides L. & Amaranthaceae & M \\
\hline 69 & Palo de sangre & Nagü Ne & Brosimum guianense (Aubl) Huber & Moraceae & $\begin{array}{l}\text { C, Art, } \\
\mathrm{H}, \mathrm{M}\end{array}$ \\
\hline 70 & Palisangre & Pucüre & Brosimum rubescens Taubert & Moraceae & M \\
\hline 71 & Pan del árbol & Paũ chane & Artrocarpus altilis (Parkinson)Fosberg & Moraceae & A \\
\hline 72 & Papaya* & Poxpayu & Carica papaya Linn & Caricaceae & A \\
\hline 73 & Parinari & Ngumari & Couepia chrysocalyx (Poepp.)Benth \& Hook.f. & Chrysobalanaceae & A \\
\hline 74 & Pichirina & Pexpe & Vismia angusta Miquel. & Hypericaceae & C, Art \\
\hline 75 & Pijuayo* & Ĩxtü & Bactris gasipaes H.B.K & Arecaceae & $\mathrm{A}, \mathrm{T}$ \\
\hline 76 & Piñon* & Piãũ & Jatropha curcas L. & Euphorbiaceae & M \\
\hline 77 & Pomarrosa* & Maãme & Syzygium malacense (L) & Myrtaceae & A \\
\hline 78 & Pona & Yura & Iriartea deltoidea R.\&P. & Arecaceae & $\mathrm{C}, \mathrm{M}$ \\
\hline 79 & Remocaspi & Nomane & Aspidosperma excelsum Bentham & Apocynaceae & M \\
\hline 80 & Renaco & Paxinü & Ficus guianensis Desvaus & Moraceae & M \\
\hline 81 & Retama & - & Senna alata (L.) Roxburgh & Fabaceae & M \\
\hline 82 & Sacha mango* & Mangua chicü & Grias neuberthii J. F Macbride & Lecythidaceae & $\mathrm{A}$ \\
\hline 83 & Sanango & Chanagu & Faramea anisocalyx Poeppig & Rubiaceae & M \\
\hline 84 & Sandia* & Worachia & Citrullus lanatus (Thumb)Matsum\& Nakai. & Cucurbitaceae & $\mathrm{A}$ \\
\hline 85 & Sangre de grado* & Cuxmaca & Croton lechleri Muell Arg. & Euphorbiaceae & M \\
\hline 86 & Shapaja & - & Attalea huebneri (Burret) Zona & Arecaceae & $\mathrm{C}$ \\
\hline 87 & Shebon & Moru & Attalea bassleriana (Burret) Zona & Arecaceae & $\mathrm{C}, \mathrm{M}$ \\
\hline 88 & Shimbillo & Unawachu cauré & Inga nobilis Willd & Fabaceae & $\mathrm{A}, \mathrm{Comb}$ \\
\hline 89 & Shiringa & Chiriüga & Hevea guianensis Aublet & Euphorbiaceae & $\mathrm{C}$ \\
\hline 90 & Suelda con suelda & Muyanü & Phthirusa pyrifolia (Kunth) Eicheler & Loranthaceae & M \\
\hline 91 & Tambishi & Tuü & Heteropsis linearis A.C. Sm. & Araceae & C, Art \\
\hline 92 & Tamshi Alambre & Õxü & Heteropsis flexuosa Kunth & Araceae & Art \\
\hline 93 & Tornillo & Túx & Cedrelinga cateniformis (Ducke) Ducke & Fabaceae & $\mathrm{C}$ \\
\hline 94 & Toronja* & Irimawa & Citrus maxima (J.Burman) Merrill & Rutaceae & $\mathrm{A}, \mathrm{M}$ \\
\hline 95 & Ubos* & Yomeru & Spondias mombin Linn & Anacardiaceae & $\mathrm{A}, \mathrm{M}$ \\
\hline 96 & Ungurahui* & Düx & Oneocarpus bataua C. Martius & Arecaceae & $\mathrm{A}, \mathrm{C}$ \\
\hline 97 & Uña de gato & Michi patü & Uncaria guianensis (Aublet) Gmelin & Rubiaceae & $\mathrm{M}$ \\
\hline 98 & Uvilla* & Chixã & Pourouma cecropiifolia Mart. & Cecropiaceae & A \\
\hline 99 & Yahuar piri piri* & Chuxpi & Eleutherine bulbosa (Miller) Urban & Iridaceae & M \\
\hline 100 & Yarina & Ngumüchi & Phytelephas macrocarpa R.\& P. & Arecaceae & $\mathrm{A}, \mathrm{C}$ \\
\hline 101 & Yuca* & Túe & Manihot esculenta Crantz & Euphorbiaceae & A \\
\hline
\end{tabular}

A: Alimenticio, M: Medicinal, C: Construcción, Comb: Combustible, Art: Artesanía, T: Tintes, H: Herramienta *: Especies cultivadas. 
Tabla 2. Usos medicinales de las especies vegetales

\begin{tabular}{|c|c|c|c|c|c|c|}
\hline $\mathbf{N}^{\circ}$ & $\begin{array}{l}\text { Nombre } \\
\text { común }\end{array}$ & Nombre científico & Usos & $\begin{array}{l}\text { Parte } \\
\text { utilizada }\end{array}$ & Preparación & Aplicación \\
\hline 1 & Ají & Capsicum annuиm $\mathrm{L}$. & Antimalárico y amigdalitis & Hojas y frutos & Infusión & Oral \\
\hline 2 & Algodón & Gossypium barbadense L. & Tratar el frío & Hojas y flores & Infusión & $\begin{array}{l}\text { Ligadas (baños } \\
\text { de vapor) }\end{array}$ \\
\hline 3 & Amasisa & Erythrina fusca Lour. & $\begin{array}{l}\text { Tumores, inflamación y } \\
\text { cáncer }\end{array}$ & Corteza & Cocimiento & $\begin{array}{l}\text { Oral y } \\
\text { emplastos }\end{array}$ \\
\hline 4 & Arazá & Eugenia stipitata subsp stipitata Mc. Vaugh & Cáncer & Frutos & Directo & Oral \\
\hline 5 & Arroz & Oryza sativa $\mathrm{L}$. & Cáncer & semillas & Directo & oral \\
\hline 6 & Caballusa & Triumfetta althaeoides Lamarck & Diarrea & hojas & Infusión & Oral \\
\hline 7 & Caguena & Ayapana triplinervis (Vahl) R.M.King \& H.Rob. & Afecciones respiratorias & hojas & Extracto & oral \\
\hline 8 & Cetico & Cecropia membranacea Trécul & Reumatismo & cogollo & Infusión & oral \\
\hline 9 & Chambira & Astrocaryum chambira Burret & Gastritis & $\begin{array}{l}\text { Agua del } \\
\text { fruto }\end{array}$ & Directo & oral \\
\hline 10 & Chancapiedra & Phyllanthus niruri L. & $\begin{array}{l}\text { Fiebre, riñones, hígado, } \\
\text { vesícula, cálculos }\end{array}$ & Hojas & Cocimiento & $\begin{array}{l}\text { Oral, agua de } \\
\text { tiempo }\end{array}$ \\
\hline 11 & Chiricsanango & Brunfelsia grandiflora D.Don & $\begin{array}{l}\text { Dolores musculares y } \\
\text { reumatismo }\end{array}$ & Raíces & Cocimiento & Oral \\
\hline 12 & Chontaquiro & $\begin{array}{l}\text { Diplotropis purpurea var. leptophylla } \\
\text { (Kleinhoonte)Amshoff }\end{array}$ & Amigdalitis & Hojas & Infusión & Oral \\
\hline 13 & Copaiba & Capaifera paupera (Herzog) Dwyer & Gastritis, inflamaciones & Aceite & Directo & Oral y tópica \\
\hline 14 & $\begin{array}{l}\text { Frejol o } \\
\text { Chiclayo }\end{array}$ & Vigna unguiculata (L.) Walpers & Diurético, laxante & Semillas & Cocimiento & Oral \\
\hline 15 & $\begin{array}{l}\text { Guineo } \\
\text { (plátano) }\end{array}$ & Musa paradisiaca $\mathrm{L}$. & Enfermedades del vientre & fruto & Cocimiento & Oral \\
\hline 16 & Huacapurana & Campsiandra angustifolia Spruce ex Bentham & $\begin{array}{l}\text { Diarrea, dolor de vientre, } \\
\text { hígado, reumatismo }\end{array}$ & Corteza & Cocimiento & Oral \\
\hline 17 & Huaruma & Delostoma integrifolium D.Don & $\begin{array}{l}\text { Protección de la piel, } \\
\text { proteger el cabello }\end{array}$ & hojas & Infusión & Tópica \\
\hline 18 & Huito & Genipa americana $\mathrm{L}$. & Antiparasitario & semillas & infusión & Oral \\
\hline 19 & Icoja & Unonopsis spectabilis Diels & Gastritis & corteza & Infusión & Oral \\
\hline 20 & Ipururo & $\begin{array}{l}\text { Alchornea castaneifolia (Humb. \& Bonpl. ex } \\
\text { Willd.) A. Juss. }\end{array}$ & Reumatismo & corteza & Cocimiento & Oral \\
\hline 21 & Jergón sacha & Dracontium loretense K.Krause & Mordedura de serpiente & Tubérculos & Cocimiento & Oral \\
\hline 22 & Macambo & Theobroma bicolor Bonpl. & Dolor de cabeza, fiebre & hojas & Infusión & Oral \\
\hline 23 & Malva & Malachra radiata (L.) L. & $\begin{array}{l}\text { Infección urinaria, úlcera, } \\
\text { desinflamante, sobreparto }\end{array}$ & $\begin{array}{l}\text { Hoja, raíz, } \\
\text { flor }\end{array}$ & $\begin{array}{l}\text { Chapeo y } \\
\text { cocimiento }\end{array}$ & $\begin{array}{l}\text { Aplicación } \\
\text { directa y oral }\end{array}$ \\
\hline 24 & Mango & Mangifera indica L. & $\begin{array}{l}\text { Limpieza del estómago } \\
\text { (purgar) }\end{array}$ & Semillas & Infusión & Oral \\
\hline 25 & Ojé & Ficus insipida Wildenow & Hernias & Resina & Directo & Tópica \\
\hline 26 & Paico & Dysphania ambrosioides L. & $\begin{array}{l}\text { Diarrea, purgante, comezón, } \\
\text { gastritis, desinflamante }\end{array}$ & Hoja & $\begin{array}{l}\text { Chapeo y } \\
\text { cocimiento }\end{array}$ & $\begin{array}{l}\text { Aplicación } \\
\text { directa y oral }\end{array}$ \\
\hline 27 & Palisangre & Brosimum rubescens Taubert & $\begin{array}{l}\text { Cáncer de vientre, } \\
\text { esterilizar a la mujer }\end{array}$ & Palo o tronco & Cocimiento & Oral \\
\hline 28 & Palo de sangre & Brosimum guianense (Aubl) Huber & $\begin{array}{l}\text { Cáncer de vientre, } \\
\text { esterilizar a la mujer }\end{array}$ & Palo o tronco & Cocimiento & Oral \\
\hline 29 & Piñón & Jatropha curcas L. & $\begin{array}{l}\text { Alcoholismo, patico, } \\
\text { gastritis, heridas, fiebre }\end{array}$ & $\begin{array}{l}\text { Semillas, } \\
\text { resina }\end{array}$ & Directo & Oral , tópica \\
\hline 30 & Pona & Iriartea deltoidea R.\&P. & $\begin{array}{l}\text { Limpieza del estómago y } \\
\text { curar miedo en los niños }\end{array}$ & semillas & Directo & Oral \\
\hline 31 & Remocaspi & Aspidosperma excelsum Bentham & Antiparasitario & corteza & Infusión & Oral \\
\hline 32 & Renaco & Ficus guianensis Desvaus & $\begin{array}{l}\text { Reumatismo, fractura de } \\
\text { huesos }\end{array}$ & resina & Directo & Tópica \\
\hline 33 & Retama & Senna alata (L.) Roxburgh & $\begin{array}{l}\text { Antibacteriano, diarreas, } \\
\text { infecciones }\end{array}$ & Hojas, flor & Infusión & Oral \\
\hline 34 & Sanango & Faramea anisocalyx Poeppig & $\begin{array}{l}\text { Para que los niños sean } \\
\text { fuertes }\end{array}$ & corteza & Infusión & Oral \\
\hline 35 & $\begin{array}{l}\text { Sangre de } \\
\text { grado }\end{array}$ & Croton lechleri Muell Arg. & Cicatrizante & resina & Directo & Tópica \\
\hline
\end{tabular}


Tabla 2. Continuación

\begin{tabular}{|c|c|c|c|c|c|c|}
\hline $\mathbf{N}^{\circ}$ & $\begin{array}{l}\text { Nombre } \\
\text { común }\end{array}$ & Nombre científico & Usos & $\begin{array}{l}\text { Parte } \\
\text { utilizada }\end{array}$ & Preparación & Aplicación \\
\hline 36 & Shebon & Attalea bassleriana (Burret) Zona & Tratar las fracturas & frutos & Directo & Tópica \\
\hline 37 & $\begin{array}{l}\text { Suelda con } \\
\text { suelda }\end{array}$ & Phthirusa pyrifolia (Kunth) Eicheler & Bronquios y fracturas & Hojas & Directo & Tópica \\
\hline 38 & Toronja & Citrus maxima (J.Burman) Merrill & Reumatismo & corteza & Directo & Tópica \\
\hline 39 & Ubos & Spondias mombin Linn & Lavado de la piel infectada & corteza & Directo & Tópica \\
\hline 40 & Uña de gato & Uncaria guianensis (Aubl.) J.F. Gmel. & $\begin{array}{l}\text { Gastritis, cáncer, próstata, } \\
\text { lavar, heridas, hígado, dolor } \\
\text { de muela y cuerpo }\end{array}$ & $\begin{array}{l}\text { Corteza, raíz, } \\
\text { hojas }\end{array}$ & Cocimiento & Oral \\
\hline 41 & $\begin{array}{l}\text { Yahuar piri } \\
\text { piri }\end{array}$ & Eleutherine bulbosa (Mill.) Urb. & Diarrea, dolores musculares & Bulbos & Infusión & Oral \\
\hline
\end{tabular}

Tabla 3. Relación de usos por familias de especies vegetales

\begin{tabular}{|c|c|c|c|c|c|c|c|c|}
\hline $\mathrm{N}^{\circ}$ & Familias & Alimentación & Artesanía & Combustible & Construcción & Herramienta & Medicinal & Tinte \\
\hline 1 & Amaranthaceae & & & & & & 1 & \\
\hline 2 & Anacardiaceae & 2 & & & & & 2 & \\
\hline 3 & Annonaceae & 2 & & & 1 & & 1 & \\
\hline 4 & Apocynaceae & 1 & & & 3 & & 1 & \\
\hline 5 & Araceae & 1 & 2 & & 1 & & 1 & \\
\hline 6 & Arecaceae & 8 & 1 & & 7 & 2 & 3 & 2 \\
\hline 7 & Asteraceae & & & & & & 1 & \\
\hline 8 & Bignoniaceace & & & & & 1 & 1 & \\
\hline 9 & Bixaceae & & & & & & & 1 \\
\hline 10 & Burseraceae & & & & 1 & & & \\
\hline 11 & Caricaceae & 1 & & & & & & \\
\hline 12 & Cecropiaceae & 1 & & & 1 & & 1 & \\
\hline 13 & Chrysobalanaceae & 1 & & & & & & \\
\hline 14 & Clusiaceae & 1 & & & & & & \\
\hline 15 & Cucurbitaceae & 1 & & & & & & \\
\hline 16 & Euphorbiaceae & 2 & & & 2 & & 4 & \\
\hline 17 & Fabaceae & 4 & 2 & 1 & 4 & & 6 & \\
\hline 18 & Hypericaceae & & 1 & & 1 & & & \\
\hline 19 & Iridaceae & & & & & & 1 & \\
\hline 20 & Lauraceae & & & & 2 & & & \\
\hline 21 & Lecythidaceae & 2 & & & 1 & & & \\
\hline 22 & Loranthaceae & & & & & & 1 & \\
\hline 23 & Malvaceae & 1 & & & & & 3 & \\
\hline 24 & Meliaceae & & & & 2 & & & \\
\hline 25 & Moraceae & 1 & 2 & & 1 & 1 & 4 & \\
\hline 26 & Musaceae & 1 & & & & & 1 & \\
\hline 27 & Myrtaceae & 3 & & & & & 1 & \\
\hline 28 & Olacaceae & & 1 & & 1 & & & \\
\hline 29 & Passifloraceae & 1 & & & & & & \\
\hline 30 & Poaceae & 3 & & & & & 1 & \\
\hline 31 & Pteridaceae & 1 & & & & & & \\
\hline 32 & Rubiaceae & 1 & & & 1 & & 3 & 1 \\
\hline 33 & Rutaceae & 3 & & & & & 1 & \\
\hline 34 & Sapotaceae & 1 & & & & & & \\
\hline 35 & Simaroubaceae & & & & 1 & & & \\
\hline 36 & Solanaceae & 1 & & & & & 2 & \\
\hline 37 & Sterculiaceae & 1 & & & & & & \\
\hline 38 & Tiliaceae & & & & & & 1 & \\
\hline 39 & Voshysiaceae & & & & 1 & & & \\
\hline \multirow[t]{2}{*}{40} & Zingiberaceae & 1 & & & & & & 1 \\
\hline & & 46 & 9 & 1 & 31 & 4 & 41 & 5 \\
\hline
\end{tabular}


Tabla 4. Especies de animales usadas por los pobladores de la comunidad Tikuna de Cushillo Cocha

\begin{tabular}{|c|c|c|c|c|c|}
\hline $\mathbf{N}^{\circ}$ & Nombre común & Nombre Ticuna & Nombre científico & Familia & Usos \\
\hline \multicolumn{6}{|l|}{ Aves } \\
\hline 1 & Coro coro & Puruna & Mesembrinibis cayennensis & Threskiornithidae & A \\
\hline 2 & Espatula rosada & Tuyuyu & Ajaia ajaja & Threskiornithidae & A \\
\hline 3 & Garza blanca chica & Wocara I Ixraü & Egretta tula & Ardeidae & A \\
\hline 4 & Graza blanca chica & Wocara & Casmerodius albus & Ardeidae & A \\
\hline 5 & Garza cebra & Chuxcu & Zebrilusum dulatus & Ardeidae & A \\
\hline 6 & Garza ceniza & Cowa & Cocoi heron & Ardeidae & A \\
\hline 7 & Gavilán picudo & Ĩyü & Chondrohierax uncinatus & Accipitridae & A \\
\hline 8 & Guamayo azul y amarillo & Chaxcra & Ara ararauna & Psittacidae & V, Msc, Art \\
\hline 9 & Guacamayo escarlata & Nohuy & Ara macao & Psittacidae & Msc, Art \\
\hline 10 & Guacamayo rojo & Ngoxü & Ara chloroptera & Psittacidae & $\mathrm{V}, \mathrm{Msc}, \mathrm{Art}$ \\
\hline 11 & Huapaya & Poxta & Cochlearius cochlearius & Ardeidae & A \\
\hline 12 & Loro cabeza azul & Ãiwe & Pionus menstrus & Psittacidae & Msc \\
\hline 13 & Loro frente amarilla & Powaru & Amazona ochrocephala & Psittacidae & A \\
\hline 14 & Manshaco & Yaguri & Mycteria americana & Ciconiidae & A \\
\hline 15 & Maracana & Tere & Primolius maracaná & Psittacidae & Msc, $\mathrm{V}$ \\
\hline 16 & Martin pescador & Puruna & Ceryle torquatus & Alcenidinidae & A \\
\hline 17 & Pájaro del aguaje & Caxure & Icterus chrysocephalatus & Icteridae & A \\
\hline 18 & Panguana & Topatawa & Crypturellus undulatus & Tinamidae & A \\
\hline 19 & Pato media luna & Putiri & Ana discors & Anatidae & A \\
\hline 20 & Perdiz azul & Nguga & Tinamus tao & Tinamidae & A \\
\hline 21 & Perdiz de monte & Nguga & Tinamus major & Tinamidae & A \\
\hline 22 & Pihuicho ala blanca & Exxne choxpeatü & Botrogeis versicolorus & Psittacidae & A \\
\hline 23 & Puma garza & Ngaxua & Tigrosoma lineatun & Ardeidae & A \\
\hline 24 & Ruiseñor & Puchui & Icterus icterus & Icteridae & Msc \\
\hline 25 & Sacha pato & Patu chicü & Cairina moscata & Anatidae & A \\
\hline 26 & Shansho & Ñgexnü & Opisthocomus hozin & Opisthocomidae & A \\
\hline 27 & Tanrirlla & Yoru & Eurypygas elia & Eurypygiade & Art \\
\hline 28 & Trogon pecho amarillo & Cucuna & Trogon viridis & Trogonidae & $\mathrm{A}, \mathrm{Msc}$ \\
\hline 29 & Trogon violeta & Cucuna yurucu & Trogon violaceus & Trogonidae & A \\
\hline 30 & Tucán de cuvier & Taxu & Ramphastos cuvieri & Ramphastidae & Msc \\
\hline 31 & Tucaneta anaranjada & Paiyü & Pteroglossus inscriptus & Ramphastidae & Msc \\
\hline 32 & Tucaneta parda & Paiyü & Pteroglossus castanotis & Ramphastidae & Msc \\
\hline 33 & Tumuy tumuy & Tumui & Pilherodius pileatus & Ardeidae & A \\
\hline 34 & Tuqui tuqui & Choxre & Jacana jacana & Jacanidae & Art \\
\hline 35 & Tuyuyo & Yaguri & Jabiru mycteria & Ciconiidae & A \\
\hline 36 & Urcututu & Murucutu & Megaspops choliba & Strigidae & A \\
\hline \multicolumn{6}{|c|}{ Maníferos } \\
\hline 37 & Achuni & Chaxtü & Nasua nasua & Procyonidae & $\mathrm{M}$ \\
\hline 38 & Añuje & Chigu & Daspycrocta fuliginosa & Dasyproctidae & A \\
\hline 39 & Carachupa & Ĩcü & Dasypus novemcinctus & Daspodidae & A \\
\hline 40 & Chosna & Tox & Potos flavus & Procyonidae & A \\
\hline 41 & Fraile & Maxicu & Saimiris boliviensis & Cebidae & A \\
\hline 42 & Huangana & Nguxû́ & Tayassu pecarri & Tayassuidae & A \\
\hline 43 & Huapo colorado & Wacari & Cacajao calvus & Callitrichidae & Msc \\
\hline 44 & Huapo negro & Puüxü & Pithecia monachus & Cebidae & A \\
\hline 45 & Intipelejo & Ure & Cyclopes didactylus & Cyclopedadae & Msc \\
\hline 46 & Leoncito & Chiriri-chixma & Cebuella pygmaea & Callitrichidae & Msc, V \\
\hline 47 & Lobo de río & Airumacachixi & Pteronura brasiliensis & Mustelidae & A \\
\hline 48 & Machín blanco & Toxü & Cebus albifrons & Cebidae & Msc \\
\hline 49 & Machín negro & Taicüre & Cebus apella & Cebidae & Msc \\
\hline 50 & Majas & Nga & Agouti paca & Agoutidae & A \\
\hline 51 & Mono choro & Õme & Lagothrix lagotricha & Cebidae & A \\
\hline 52 & Nutria & Aixtü & Lontra longicaudis & Mustelidae & A \\
\hline 53 & Otorongo & $\mathrm{Ai}$ & Panthera onca & Felidae & $\mathrm{V}$ \\
\hline 54 & Pelejo & Powi & Bradypus variegatus & Bradypodidae & M \\
\hline 55 & Pelejo de altura & Woxē-base & Choloepus didactylus & Megalonychidae & Msc \\
\hline 56 & Perro del monte & $\begin{array}{l}\text { Airu i dauchitacax cuai } \\
\text { cuai }\end{array}$ & Eira barbara & Mustelidae & Msc \\
\hline 57 & Pichico labiado & Yaxri & Saguinus labiatus & Callitrichidae & Msc \\
\hline
\end{tabular}


Tabla 4. Continuación

\begin{tabular}{|c|c|c|c|c|c|}
\hline $\mathbf{N}^{\circ}$ & Nombre común & Nombre Ticuna & Nombre científico & Familia & Usos \\
\hline 58 & Pichico pardo & Yaxri & Saguinus fucicollis & Callitrichidae & Msc, V \\
\hline 59 & Puerco espín & Ñaxi & Coendou bicolor & Erethizontidae & Art \\
\hline 60 & Puma colorado & Ngexma & Puma concolor & Felidae & $\mathrm{V}$ \\
\hline 61 & Rata del monte & úxca & Oryzomys bolivaris & Cricetidae & A \\
\hline 62 & Ronsoco & Copiwara & Hydrochaerishy drochaeris & Hydrochaeridae & $\mathrm{A}, \mathrm{V}$ \\
\hline 63 & Sacha vaca & Nacü & Tapirus terrestris & Tapiridae & $\mathrm{A}, \mathrm{V}$ \\
\hline 64 & Sajino & Ngaxwü & Tayassu tajacu & Tayassuidae & A \\
\hline 65 & Tigrillo & Queture & Leopardus pardalis & Felidae & V \\
\hline 66 & Tocón colorado & Duare & Callicebus cupreus & Pitheciidae & Msc \\
\hline 67 & Tocón negro & Duare & Callicebus torquatus & Pitheciidae & Msc \\
\hline 68 & Vaca marina & Airuwe & Trichechus inunguis & Trichechidae & A \\
\hline 69 & Venado cenizo & Yawe & Mazama gouazoubira & Cervidae & $\mathrm{A}, \mathrm{V}$ \\
\hline 70 & Venado colorado & Cowü & Mazama americana & Cervidae & $\mathrm{A}, \mathrm{V}$ \\
\hline \multicolumn{6}{|c|}{ Anfibios y reptiles } \\
\hline 71 & Anaconda & Tuxchinawe & Eunectes murinis & Boidae & $\mathrm{V}$ \\
\hline 72 & Boa negra & Yoxi & Boa constrictor & Boidae & $\mathrm{M}, \mathrm{V}$ \\
\hline 73 & Boa esmeralda/Loro machaco & Caxcu & Corallus caninus & Boidae & V \\
\hline 74 & Cupiso & - & Podocnemis sextuberculata & Podocnemididae & A \\
\hline 75 & Dirin dirin & Curua & Paleosuchus palpebrosus & Alligatoridae & $\mathrm{A}, \mathrm{M}$ \\
\hline 76 & Iguana cabeza roja & Ngiri & Dracaena guianensis & Teiidae & $\mathrm{A}, \mathrm{V}$ \\
\hline 77 & Lagarto blanco & Yoca i choxú & Caiman crocodilus & Alligatoridae & $\mathrm{A}, \mathrm{M}, \mathrm{V}$ \\
\hline 78 & Lagarto negro & Coya i waüú́ & Melanosuchus niger & Alligatoridae & M \\
\hline 79 & Matamata & Ngaiyare & Chelus fimbriatus & Chelidae & $\mathrm{A}, \mathrm{M}$ \\
\hline 80 & Motelo & Ngoxbü & Geochelone denticulata & Testudinidae & $\mathrm{A}, \mathrm{M}$ \\
\hline 81 & Sapo hualo & Curucu & Rhinella marina & Bufonidae & A \\
\hline 82 & Charapa & - & Podocnemis expansa & Podocnemididae & $\mathrm{A}, \mathrm{M}$ \\
\hline 83 & Iguana verde & - & Iguana iguana & Iguanidae & A \\
\hline 84 & Iguana cabeza negra & - & Tupinambis teguixin & Teiidae & A \\
\hline \multicolumn{6}{|c|}{ Peces } \\
\hline 85 & Acaharazú & Oxcara & Astronotus ocellatus & Cichlidae & A \\
\hline 86 & Anguila & Taxu & Electrophorus electricus & Elecophoride & $\mathrm{O}$ \\
\hline 87 & Arahuana & Orawana & Osteoglossum bicirrhosum & Ossteoglossidae & A \\
\hline 88 & Ashara & Yapexewa & Leiarius marmoratus & Pimelodidae & $\mathrm{O}$ \\
\hline 89 & Atinga & Peraxi & Synbranchus marmoratus & Symbranchidae & $\mathrm{O}$ \\
\hline 90 & Bagre & Moni & Microglanis poecilus & Pimelodidae & A \\
\hline 91 & Bocón & Doxma & Ageneiosus brevifilis & Ageneiosidae & A \\
\hline 92 & Boquichico & Chirimata_caxweya & Prochilodus nigricans & Prochilodontidae & A \\
\hline 93 & Bujurqui & Chúná & Chaetobranchus flavescens & Cichlidae & A \\
\hline 94 & Bujurqui punta shimi & Woxpana & Satanoperca jurupari & Cichlidae & $\mathrm{O}, \mathrm{A}$ \\
\hline 95 & Cahuara & Wocu & Pterodoras granlosus & Doradidae & A \\
\hline 96 & Canero azul & Coneru & Cetopsis coecutiens & Cetopsidae & $\mathrm{O}$ \\
\hline 97 & Carachama & Owaru & Monistiancistrus carachama & Loricaridae & A \\
\hline 98 & Carachama barbuda & Chaüna & Ancistrus dolichopterus & Loricaridae & A \\
\hline 99 & Carachama común & Owaru ixrä̈ (Woxmaru) & Lyposarcus pardalis & Loricaridae & A \\
\hline 100 & Chambira & Wainayu & Rhaphiodon vulpinus & Cynodontidae & A \\
\hline 101 & Corvina & Tücüena & Plagioscion squasissimus & Sciaenidae & A \\
\hline 102 & Curuhuara & Chüwü & Myleus rubripinnis & Serrasalmidae & A \\
\hline 103 & Doncella & Yuta & Pseudoplatystoma fascciatum & Pimelodidae & A \\
\hline 104 & Dorado & Yuta taxü & Brachyplatystoma rousseauxii & Pimelodidae & A \\
\hline 105 & Fasaco & Dewara & Hoplias malabaricus & Erythinidae & A \\
\hline 106 & Gamitana & Tomacachi & Colossoma macropomun & Serrasalmide & A \\
\hline 107 & Huapeta & - & Hydroycus scombenroides & Cynodontidae & A \\
\hline 108 & Lisa & Chirimata-Caweya & Schizodon fasciata & Anostomidae & A \\
\hline 109 & Lisa cachete colorado & Oxta & Leoporinus tridasciatus & Anostomidae & A \\
\hline 110 & Lisa leporinus & Waracu & Leoporinus fasciculata & Anostomidae & A \\
\hline 111 & Llambina & Yowarachi & Potamorhina altamazonica & Curimatidae & A \\
\hline 112 & Maparate & Mapara & Hypophthalmus edentatus & Hypophthalmidae & A \\
\hline 113 & Maparateleguia & Mapara & Auchenipterus nuchalis & Auchenipteridae & A \\
\hline 114 & Mojara & Matupiri & Astyanax fasciculatus & Characidae & A \\
\hline 115 & Mota & Muta & Callophysus macropterus & Pimelodidae & A \\
\hline
\end{tabular}


Tabla 4. Continuación

\begin{tabular}{|c|c|c|c|c|c|}
\hline $\mathbf{N}^{\circ}$ & Nombre común & Nombre Ticuna & Nombre científico & Familia & Usos \\
\hline 116 & Paco & Pocu & Piractus brachypomus & Pimelodidae & A \\
\hline 117 & Paiche & Dexchi & Arapaima gigas & Arapaimidae & A \\
\hline 118 & Palometa & Pacu & Mylosso maduriventris & Serrasalmidae & A \\
\hline 119 & Panga raya & Oromacha & Achirus achirus & Achiridae & $\mathrm{O}$ \\
\hline 120 & Paña larga & $\begin{array}{l}\text { Uchuma i maxchiwexü } \\
\text { aixcüma }\end{array}$ & Serrasalmus elongatus & Serrasalmidae & A \\
\hline 121 & Paña roja & Uchuma i daxũ & Serrasalmus natteri & Serrasalmidae & A \\
\hline 122 & Pez ángel & - & Pterophyllum scalare & Cichlidae & $\mathrm{O}$ \\
\hline 123 & Pez churero & Chureru & Megalodoras irwini & Doradidae & $\mathrm{O}, \mathrm{A}$ \\
\hline 124 & Pez globo & - & Colomesus asellus & Symbranchidae & $\mathrm{O}$ \\
\hline 125 & Pez hoja & Choxni a naxiatüraü & Monocirrhus polyacanthus & Nandidae & $\mathrm{O}$ \\
\hline 126 & Pez torre & Puxã & Phracocephalus hemiliopterus & Pimelodidae & $\mathrm{O}, \mathrm{A}$ \\
\hline 127 & Ractacara & Yowarachi taichipaü & Curimata vittata & Curimatidae & A \\
\hline 128 & Raya motoro & - & Potamotrygon motoro & Potamotrygonidae & $\mathrm{O}$ \\
\hline 129 & Rego rego & Cuxyu chicü & Agamysis albomaculatus & Doradidae & A \\
\hline 130 & Rego rego & Cuxyu chicü & Amblidoras hancocki & Doradidae & A \\
\hline 131 & Sábalo cola negra & Eruma & Brycon elanopterus & Characidae & A \\
\hline 132 & Sábalo cola roja & Ngexchi & Brycon cephalus & Characidae & A \\
\hline 133 & Sardina & Arawiri & Triportheus elongatus & Characidae & A \\
\hline 134 & Shiripira & úxre & Sorubim lima & Pimelodidae & $\mathrm{O}$ \\
\hline 135 & Shiruy & Chirui & Corydoras napoensi & Callichthydae & A \\
\hline 136 & Shitari & - & Loricarrichthys maculatus & Loricaridae & A \\
\hline 137 & Shuyo & Oxü & Hoplerythrinu sunitaeniatus & Erythrinidae & A \\
\hline 138 & Tigre zúngaro & Yuta a aimatüraü & Pseudoplatystoma tigrinum & Pimelodidae & A \\
\hline 139 & Toa & Coxchi & Hemisorubim platyrhynchos & Pimelodidae & A \\
\hline 140 & Tucunare & Tucunari & Cichla monoculos & Cichlidae & A \\
\hline 141 & Turushuqui & Turuchuki & Oxydoras niger & Doradidae & A \\
\hline 142 & Yahuarachi & Yoarachi & Potamorhina latior & Curimatidae & A \\
\hline 143 & Yaraquí & Yáxi & Semaprochilodus theraponura & Prochilodontidae & A \\
\hline 144 & Yulilla & Yurilla & Anotus elogantus & Hemiodontidae & A \\
\hline 145 & Zúngaro alianza & Yuta & Brachyplatystoma juruense & Pimelodidae & $\mathrm{O}$ \\
\hline 146 & Zúngaro tabla barba & Coxtu & Goslinia platynema & Pimelodidae & A \\
\hline
\end{tabular}

A: Alimento; O: Ornamental; Msc: Mascota; M: Medicinal; V: Venta; Art: Artesanía

Tabla 5. Uso tradicional de especies de animales utilizados como medicinales.

\begin{tabular}{|c|c|c|c|c|c|c|c|}
\hline $\mathbf{N}^{\circ}$ & $\begin{array}{l}\text { Nombre } \\
\text { común }\end{array}$ & Nombre científico & Familia & Uso tradicional & $\begin{array}{c}\text { Parte } \\
\text { utilizada }\end{array}$ & Preparación & Aplicación \\
\hline 1 & Achuni & Nasua nasua & Procyonidae & Problemas eréctiles & Pene seco & $\begin{array}{l}\text { Macerado en aguardiente } \\
\text { con siete raíces }\end{array}$ & Oral \\
\hline 2 & Boa negra & Boa constritor & Boidae & Afecciones respiratorias & Manteca & Diluida al sol & Oral \\
\hline 3 & Charapa & Podocnemis expansa & Podocnemididae & Desmamar al bebé & Manteca & $\begin{array}{l}\text { Aplica en los senos de la } \\
\text { mama }\end{array}$ & Tópica \\
\hline 4 & Dirin dirin & Paleosuchus palpebrosus & Alligatoridae & Bronquitis & Manteca & Frotación & Tópica \\
\hline 5 & Lagarto blanco & Caiman crocodilus & Alligatoridae & Bronquitis & Manteca & Diluida al sol & Oral \\
\hline 6 & Lagarto negro & Melanosuchus niger & Alligatoridae & Tuberculosis, bronquitis & Manteca & Diluida al sol & Oral \\
\hline 7 & Matamata & Chelus fimbriatus & Chelidae & Diarrea & Casco & Decocción & Oral \\
\hline 8 & Motelo & Geochelone denticulata & Testudinidae & Diarrea & Casco & Decocción & Oral \\
\hline 9 & Pelejo & Bradypus variegatus & Bradypodidae & Dolor de estómago & manteca & Diluida & Tópica \\
\hline
\end{tabular}


los usos tradicionales de las 41 especies vegetales identificadas como medicinales. En la Tabla 3 se detalla la relación entre las categorías de usos y las familias de especies vegetales.

De las 101 especies de plantas registradas, 35 son cultivadas en las chacras y huertas, las demás especies se encuentran en forma silvestre.

Con respecto a la etnozoología se registraron 146 especies de animales, distribuidas en 127 géneros y 65 familias, utilizados para diversos fines. Estas especies se clasificaron en seis categorías de uso: Alimenticio (A), Artesanía (Art.), Mascota (Msc.), Medicinal (M), Ornamental (O) y Venta (V). Se encontró 36 especies de aves, 34 de mamíferos, 14 de anfibios y reptiles, y 62 de peces (Tabla 4).

Para el caso de las especies de animales, las familias más representativas fueron, en orden decreciente y por clasificación: Aves: Ardeidae (9 especies), Psittacidae (7 especies); Mamíferos: Cebidae (5 especies), Callitrichidae (4 especies); Anfibios y reptiles: Alligatoridae (3 especies) y Boidae (3 especies); Peces: Pimelodidae (11 especies), Serrasalmidae (5 especies), Cichlidae (5 especies), Doradidae (5 especies), Loricaridae (4 especies), y Characidae (4 especies).

Las categorías de uso con mayor número de especies de animales fueron alimentación con 103 especies y mascotas con 21 especies.

Se identificaron 9 especies de animales con uso medicinal, en la Tabla 5 se detallan los usos tradicionales de estas especies.

Cabe señalar que se reportan tanto en 11 especies de flora y 9 especies de fauna que no tienen nombre en idioma Tikuna, porque los pobladores de la comunidad les denominan con su nombre en español.

\section{Discusión}

En total, la comunidad indígena Tikuna de Cushillo Cocha reporta un conocimiento sobre 101 plantas y 146 animales. De las 46 especies vegetales que se reportan como alimento, Manihot esculenta (yuca), Musa paradisiaca (plátano), Pouteria caimito (caimito), son las más representativas, siendo productos destacados dentro de su sistema alimenticio tradicional tal como los reportan Acosta y Zoria (2012) y Acosta (2001).

Respecto a las especies vegetales medicinales, existe una sola coincidencia con lo reportado por Quintana (2012) para la comunidad Tikuna colombiana de Macedonia, que es el caso de la especie Eleutherine bulbosa, (Yahuar piripiri) aunque difieren en la parte utilizada y en el uso tradicional. También existen coincidencia con los géneros Gossypium (algodón), Uncaria (uña de gato) y Cecropia (cetico) pero difieren en su uso tradicional. El uso de la Jatropha curcas (piñón blanco) también es reportado para el tratamiento de la fiebre en los Tikuna del Parque Nacional de Jaú en la Amazonía brasileña (Rodrigues 2006).

Dada la gran cantidad de especies de peces reportadas (62) se comprueba que la pesca es la actividad económica de mayor importancia entre los Tikuna, debido a la gran riqueza de este recurso en la región (Mayor \& Bodmer 2009) y las técnicas efectivas utilizadas para esta actividad que son de gran antigüedad (Vieco \& Oyuela 1999).

Según este estudio la mayoría de plantas y animales que conocen y utilizan los indígenas de la comunidad Tikuna de Cushillo
Cocha es para la alimentación, cura de sus enfermedades y para la construcción de sus viviendas. Es importante resaltar que los principales padecimientos que curan con las plantas medicinales son: tos, asma, infecciones, malaria, fiebres, gripe, artritis y como desinfectante. El aprovisionamiento de estas son el bosque y sus chacras (Acosta \& Zoria 2012).

Se ha podido constatar que los Tikuna actualmente no dependen sólo del bosque para el aprovisionamiento de plantas medicinales, sino que lo cultivan en sus chacras y huertas. Esta práctica es una estrategia que utilizan para enfrentar la problemática de que el bosque ha disminuido debido a la deforestación y que está cada vez más lejos de la comunidad y con menos diversidad de especies.

Con esto se pone de manifiesto la importancia de documentar toda la riqueza de su sabiduría ancestral utilizada de manera natural en una comunidad, como también los usos de dichas plantas y animales, contribuyendo al conocimiento científico que se genera en las etnias de la Amazonía del Perú y de esta manera poder contribuir decisivamente a los esfuerzos de conservación y manejo de estas culturas y de sus ecosistemas (La Torre-Cuadros 2008). De acuerdo con los resultados obtenidos, se comprobó que el bosque es la fuente principal para su alimentación, cura de enfermedades y otras cinco categorías más de usos con importante aceptación en la población indígena Tikuna del poblado de Cushillo Cocha.

\section{Agradecimientos}

A los pobladores y autoridades de la comunidad indígena Cushillo Cocha por su colaboración desinteresada. Al ingeniero Otoniel Mendoza, coordinador de la oficina de Caballo Cocha - IIAP y a los investigadores del PIBA, Blgo. Cesar Delgado e Ing. Agustín Gonzáles, por su contribución para el desarrollo del estudio y en las labores de campo.

\section{Literatura citada}

Acosta L.E. 2001. Los sistemas de producción de la etnia Tikuna del resguardo Nariño, sur del trapecio Amazónico: una aproximación socioeconómica. Cuadernos de Desarrollo rural 46: 101-132. <http://revistas.javeriana.edu.co/index.php/ desarrolloRural/article/view/2315> (Acceso 06/01/2016)

Acosta L.E. \& J. Zoria. 2012. Conocimientos tradicionales Tikuna en la agricultura de chagras y los mecanismos innovadores para su protección. Boletim do Museu Paraense Emílio Goeldi. Ciências Humanas 7(2): 417-433. doi: http://dx.doi. org/10.1590/S1981-81222012000200007.

Alexiades M. 1985. Proyecto Biorecursos para la creación de una red internacional de datos etnobiológicos. Primer informe sobre bancos de biodatos de Tambopata (julio-diciembre). Bioresources Limited, Londres, Inglaterra.

Alves R.R.N. \& H. Alves. 2011. The faunal drugstore: Animal-based remedies used in traditional medicines in Latin America. Journal of Ethnobiology and Ethnomedicine 7:9. http:// dx.doi.org/10.1186/1746-4269-7-9.

Bajak A. 2014. Manejo de colecciones etnobiológicas para descubrimiento y desarrollo farmacéutico en América Latina. Latín American Science dot ORG. <http://latinamericanscience. $\mathrm{org} / \mathrm{spanish} / 2014 / 01 / \mathrm{manejo-de}$-colecciones-etnobiologicas-para-descubrimiento-y-desarrollo-farmaceutico-enamerica-latina/ $\geq$ (Acceso 23/02/2016).

Brack Egg A. 1999. Diccionario enciclopédico de plantas útiles del Perú. CBC-Centro de Estudios Regionales Andinos "Bartolomé de las Casas", Cuzco, p.7.

Carlini E.A., E. Rodrigues, F.R. Mendes, R. Tabach, B. Gianfratti. 2006. Treatment of drug dependence with Brazilian herbal medicines. Brazilian Journal Pharmacognosy 16 (supl.): 690-695. http://dx.doi.org/10.1590/S0102695X2006000500016. 
Chuecas A. 2003. Lo cultural y lo político en los procesos de globalización: pueblos indígenas. En: Castro C, Cornejo M (ed). Acceso a los servicios bibliotecarios y de información en los pueblos indígenas de América Latina. Lima: Centro Amazónico de Antropología y Aplicación Práctica; Pp. 23-30.

Correa C. 2001. (en línea). Los conocimientos tradicionales y la propiedad intelectual: cuestiones y opciones acerca de la protección de los conocimientos tradicionales. <http:// www.biodiversidadla.org/Objetos_Relacionados/file_folder/ Archivos_pdf/Los_conocimientos_tradicionales_y_la_propiedad_intelectual $\geq$ (acceso 04/03/2016).

Del-Águila-Chávez J. 2013. La diversidad y riqueza de los peces Amazónicos. En M. Martín-Brañas, ed. Amazonía: Guía ilustrada de flora y fauna, Ministerio del Ambiente, Perú. Pp. 186-241.

Escobar Berón G. 2002. (en línea). Introducción al paradigma de la etnobiológicas una realidad aparte. <http://www.mailxmail. com/curso-etnociencias-yage/etnobiologia-introduccionparadigma-etnobiologia> (Acceso 05/04/2016).

Estrella E. 1995. Plantas medicinales amazónicas: realidad y perspectivas. Tratado de cooperación amazónica, Lima, Perú.

Gagliardi-Urrutia L. 2013. El fabuloso mundo de la herpetofauna amazónica. En M. Martín-Brañas, ed. Amazonía: Guía ilustrada de flora y fauna, Ministerio del Ambiente, Perú. Pp. 159-184.

Hammond D.S., P.M. Dolman \& A. R. Watkinsen. 1995. Modern Tikuna swidden-fol-low management in the Columbian Amazon: Ecologically integrating market strategies and subsistence driven economics. Human Ecology 23(3): 335356. http://dx.doi.org/10.1007/BF01190136.

INEI (Instituto Nacional de Estadística e Informática). 1993. (en línea). Perú: I Censo de Comunidades Indígenas de la Amazonía (Información preliminar). INEI, Lima, Perú. <http:// proyectos.inei.gob.pe/web/biblioineipub/bancopub/Est/ Lib0001/resumeje.htm> (Acceso 02/02/2016).

La Torre-Cuadros M.A. 2008. Ciento doce años de investigación científica sobe las etnias de la Amazonía peruana. Boletín Latinoamericano de Plantas Medicinales y el Caribe 7(3): 171-179.

López C.L. 2002. Los Tikuna frente a los procesos de nacionalización en la frontera entre Brasil, Colombia y Perú. Revista colombiana de antropología 38: 77-104.

López-Wong C. 2013. Mamíferos de la Amazonía peruana. En M. Martín-Brañas, ed. Amazonía: Guía ilustrada de flora y fauna, Ministerio del Ambiente, Perú. Pp. 128-156.

Martín M. 2009. Amazonía: Guía ilustrada de flora y fauna. AECID - Ministerio del Ambiente, Perú.
Mayor P. \& R.E. Bodmer. 2009. Pueblos indígenas de la Amazonía peruana. Ed. Centros de Estudios Teológicos de la Amazonía Peruana (CETA), Iquitos, Perú, pp. 256. <http://atlasanatomiaamazonia.uab.cat/pdfs/PueblosIndigenasAmazoniaPeruana.pdf> (Acceso 29/01/2016).

Mejía K. 2013. Las palmeras de la Amazonía Peruana. En M. MartínBrañas, ed. Amazonía: Guía ilustrada de flora y fauna, Ministerio del Ambiente, Perú. Pp. 364-392.

MINCETUR. 2009. (en línea). Comunidad nativa Tikuna Cushillo Cocha. <http://www.mincetur.gob.pe/TURISMO/ OTROS/inventario\%20turistico/Ficha.asp?cod_Ficha $=3817 \geq$ (Acceso 05/02/2016).

Montes M.E. 2001. Los nombres de las plantas, sus partes y sus espacios de crecimiento. Aproximación entnolingüística a partir de datos de la lengua Ticuna, Amazonía colombiana. Universidad Nacional de Colombia. shttp://www.bdigital. unal.edu.co/3740/2900/nombresdelasplantassuspartesysusespaciosdecrecimiento.pdf $\geq$ (Acceso 06/03/2016).

Quintana R.F. 2012. Estudio de plantas medicinales usadas en la comunidad indígena Tikuna del alto Amazonas, Macedonia. Nova 10 (18): 181-193.

Rengifo-Salgado E. 2013. Árboles y Plantas: Un paraíso verde. Diversidad y fragilidad en la Amazonía. En M. Martín-Brañas, ed. Amazonía: Guía ilustrada de flora y fauna, Ministerio del Ambiente, Perú. Pp. 276-362.

Ribeiro D. \& W.R. Wise. 1979. (en línea). Grupos étnicos de la Amazonía Peruana. Comunidades y Culturas Peruanas 13. ILV, Pucallpa, Perú. _http://www-01.sil.org/americas/peru/ pubs/ccp13.pdf $\geq$ (Acceso 22/02/2016).

Rodrigues E. 2006. Plants and animals Utilized as Medicines in the Jaú National Park (JNP), Brazilian Amazon. Phytotherapy Research 20: 378-391. http://dx.doi.org/10.1002/ptr.1866

Solis F.G. 2003. Lenguas en la Amazonía Peruana. Visual Service SRL, Lima, Perú.

Valadeau C. 2009. La vida secreta de las plantas medicinales en los pueblos Kichwa, kukama-kukamiria y tikuna. Una aproximación al conocimiento de algunas plantas de uso medicinal en la comunidad educativa de ZungaroCocha. Bulletin de I'Institut francais d'études andines 38 (1): 143-145. <http:// www.redalyc.org/articulo.oa?id=12612890014> (Acceso $18 / 01 / 2016)$

Varesse S. 1983. Los grupos étnicos de la selva peruana. En Portier B., Ed. América Latina en sus lenguas. Unesco, Monte Ávila Editores, París/Caracas, Fracia/Venezuela.

Vieco J.J. \& A. Oyuela. 1999. La pesca entre los ticuna: historia, técnica y ecosistemas. Boletín de Antropología 13 (30): 73-99. <http://users.clas.ufl.edu/caycedo/historical\%20 ecology/Amazonas/1999\%20Ticuna\%20Fishing.pdf> (Acceso 24/03/2016). 